\title{
HGTector: an automated method facilitating genome-wide discovery of putative horizontal gene transfers
}

\author{
Qiyun Zhu' ${ }^{*}$, Michael Kosoy ${ }^{2}$ and Katharina Dittmar ${ }^{1 *}$
}

\begin{abstract}
Background: First pass methods based on BLAST match are commonly used as an initial step to separate the different phylogenetic histories of genes in microbial genomes, and target putative horizontal gene transfer (HGT) events. This will continue to be necessary given the rapid growth of genomic data and the technical difficulties in conducting large-scale explicit phylogenetic analyses. However, these methods often produce misleading results due to their inability to resolve indirect phylogenetic links and their vulnerability to stochastic events.

Results: A new computational method of rapid, exhaustive and genome-wide detection of HGT was developed, featuring the systematic analysis of BLAST hit distribution patterns in the context of a priori defined hierarchical evolutionary categories. Genes that fall beyond a series of statistically determined thresholds are identified as not adhering to the typical vertical history of the organisms in question, but instead having a putative horizontal origin. Tests on simulated genomic data suggest that this approach effectively targets atypically distributed genes that are highly likely to be HGT-derived, and exhibits robust performance compared to conventional BLAST-based approaches. This method was further tested on real genomic datasets, including Rickettsia genomes, and was compared to previous studies. Results show consistency with currently employed categories of HGT prediction methods. In-depth analysis of both simulated and real genomic data suggests that the method is notably insensitive to stochastic events such as gene loss, rate variation and database error, which are common challenges to the current methodology. An automated pipeline was created to implement this approach and was made publicly available at: https:/github.com/DittmarLab/ HGTector. The program is versatile, easily deployed, has a low requirement for computational resources.

Conclusions: HGTector is an effective tool for initial or standalone large-scale discovery of candidate HGT-derived genes.

Keywords: Horizontal gene transfer, Bacterial genomes, BLAST
\end{abstract}

\section{Background}

Systematic studies have shown that horizontal gene transfer (HGT) is prevalent in prokaryotes [1-3], where it serves as an important driving force of microbial evolution [4]. HGT challenges the detection of vertical inheritance patterns in prokaryotes, and the application of conventional phylogenetic approaches to infer evolutionary history of microbial clades has seen increased limitations [4-9]. In essence, the ubiquitous nature of this process calls for the need to separate the vertical and horizontal patterns in evolutionary history of bacterial

\footnotetext{
* Correspondence: qiyunzhu@buffalo.edu; kd52@buffalo.edu

${ }^{1}$ Department of Biological Sciences, University at Buffalo, State University of New York, 109 Cooke Hall, Buffalo, NY 14260, USA

Full list of author information is available at the end of the article
}

genomes. However, this is not straightforward in practice and is especially difficult for deep historical events because the horizontally acquired genes evolved along with the recipient genomes, gradually losing the signatures of their original hosts (amelioration) [10]. Furthermore, HGTs between closely related organisms, although common, are difficult to detect because in these cases donor and recipient share common compositional and phylogenetic features. So far, multiple computational methods have been developed to facilitate HGT detection, which may be loosely categorized into three main strategies based on sequence composition, phylogenetic analysis, or best BLAST matches [11-13]. However, there appears to be poor agreement between outcomes of diverse methods, 
and comparative studies have repeatedly demonstrated that depending on method different sets of putative HGT-derived genes are identified from the same dataset, reflecting limitations in the current methodology for HGT prediction [13-15].

Given the rapid increase in available annotated genome data, and the associated computational challenge of analyzing such data, the BLAST best match method has remained a popular surrogate for first pass discovery analyses of gene histories that differ from the strict vertical pattern [16]. Specifically, this strategy is practiced by sorting BLAST hits by measures such as bit scores, an indicator of sequence similarity, and the best match organism represented by the top hit is identified for each gene [17]. If the best match is a distantly related organism, instead of one expected by vertical inheritance, then the gene is categorized as likely horizontally acquired [18] (see Additional file 1: Figure S1A, B). In practice, researchers often identify the best match using the criterion of bidirectional best hits (BBH) [19] to rule out potential paralogs. This approach has been applied in numerous studies [16,20-24]. Examples of programs featuring this approach include Pyphy [25], PhyloGenie [26], NGIBWS [27], and DarkHorse [28,29], although the latter also employs a user-definable filter threshold in combination with taxonomic scaling.

As expected, BLAST-based HGT detection has limitations. The bit score is based on sequence similarity, and provides only a rough estimate of the phylogenetic history between organisms. Also, best hits do not necessarily represent the nearest neighbors [30]. Most importantly, unexpected best hit can also be caused by reasons other than HGT, such as lack of sequence information in related organisms [15], gene loss events [31], stochastic similarity [15] as well as database error [32]. In practice, the predicted HGT candidates are often rejected by downstream phylogenetic analyses [33]. Similar to phylogenetic HGT detection methods, BLAST best match is effective in detecting recent HGT events, but shows reduced sensitivity for ancient events, when donor and recipient sequences have already diverged over the long history of evolution [34]. Additionally, merely a best match does not necessarily provide insights into the direction of gene flow.

Despite the listed issues, the best BLAST match methods make use of all sequence data available in GenBank. This sidesteps the need for manual subsampling and curation of comparative sequence data that is a challenging and time-consuming step in phylogenetic methods. This strategy is therefore likely to remain a feasible solution to explore microbial evolution in a first pass step, utilizing all of the ever increasing genomic data [35].

Considering this trend, we introduce a BLAST-based method to facilitate the detection of horizontal gene histories that aims to remedy some of the above outlined issues. This approach starts with standard all-against-all BLASTP, and is followed by an investigation of the weight distribution of all hits grouped by phylogenetically informed user defined categories (illustrated in Figures 1 and 2, Additional file 1: Figure S1). A general pattern of BLAST hit distributions (a fingerprint) of the genomes of interest is computed, and BLAST hit weights of each single gene are compared to the general fingerprint. This decreases sensitivity to stochastic disturbances. Because phylogenetic information is incorporated into this process, each resulting distribution is divided by uniquely defined cutoffs into typical and atypical gene populations. Using a combination of rules, a pool of genes that is putatively horizontally derived is reported. It is recommended that the atypical gene pool be subjected to downstream phylogenetic validation of HGT, which is implemented in this pipeline.

With this approach, the method retains all advantages of BLAST-based methods, such as efficient and exhaustive searches, which also facilitates re-analysis of data following the addition of new data. Rather than using general filter thresholds, and subsequent refining by taxonomic metrics (see LPI in DarkHorse [28]), this method combines the two steps into one, and defines unique thresholds for each hierarchical level under consideration.

In order to assess the performance and robustness of this pipeline regarding the identification of putatively HGT-derived genes, it was applied towards simulated genomic data with known HGT events under consideration of various evolutionary forces. The method was also tested on real genomic datasets from multiple organismal groups, as exemplified by Rickettsia, whose HGT patterns have been previously studied [36-41]. The consistency of these results was compared to those obtained by other methods, including sequence composition and phylogenetic approaches. Overlaps between results were investigated and discussed in the framework of technical and biological challenges behind each method.

\section{Methods}

\section{Method overview}

At the core of this approach is an all-against-all BLASTP search of the protein product of each protein-coding gene (referred to as gene hereafter) of the genome(s) of interest against the genome database. For each protein, the BLAST hits are recorded and sorted by their bit scores from high to low. The bit scores are then normalized by dividing them by the bit score of the query sequence in order to account for protein length variance, so that every hit has a normalized bit score within the $0-1$ range [21]. The organism corresponding to each hit and the taxonomic ranks of each organism are identified and recorded from the NCBI taxonomy database. 


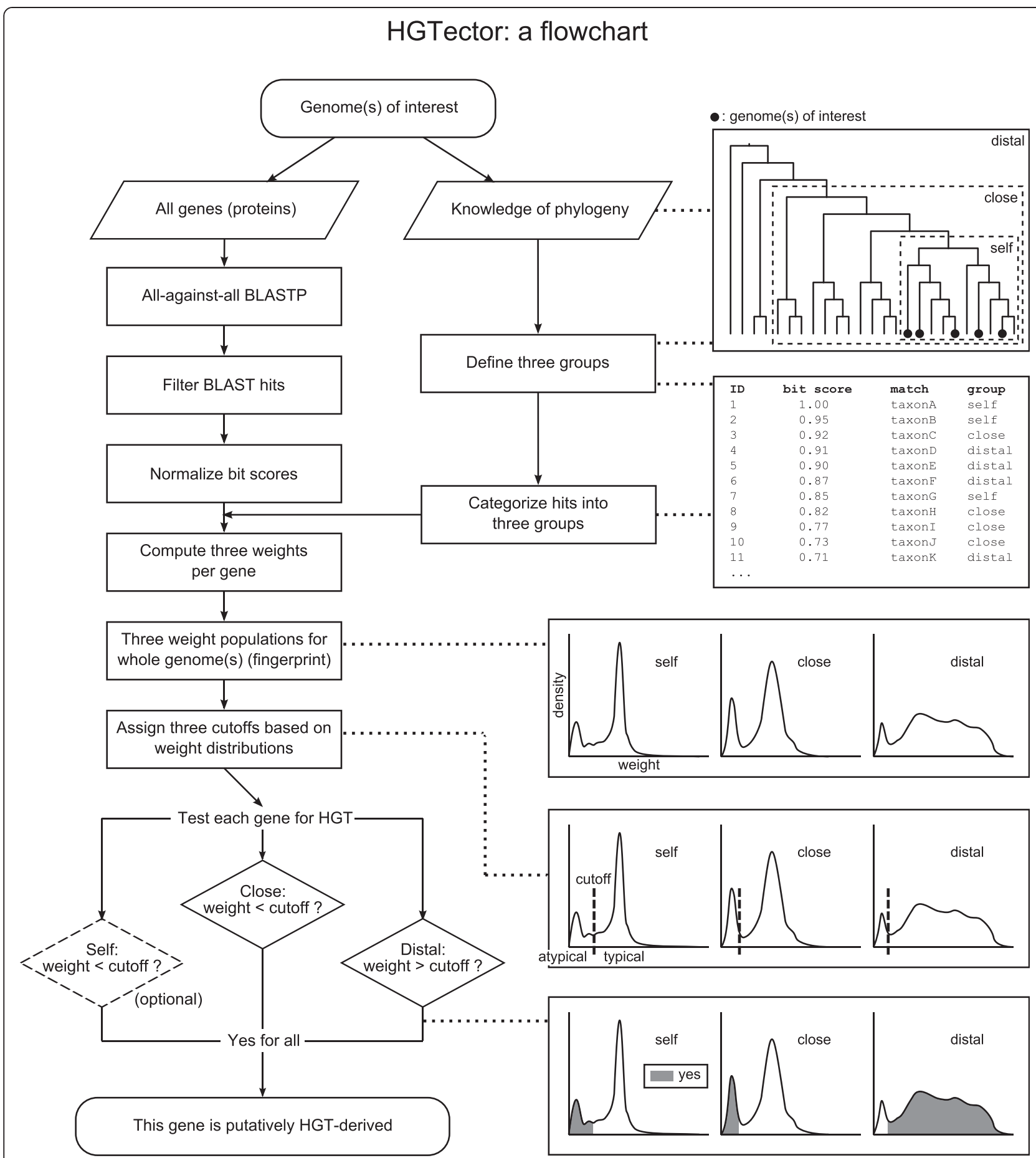

Figure 1 Flowchart illustrating the procedures of the HGTector method. Parallelograms indicate input data or information, rectangles indicate processing steps, diamonds indicate decisions, and rounded rectangles indicate start and end of the work flow. Graphic illustrations of hypothetical phylogenetic tree, BLAST hit table and fingerprint are drawn on the right side of their corresponding steps.

To proceed with the analyses, hits of each gene have to be divided into self, close and distal groups. In other words, the pipeline doesn't use phylogenetic trees or taxonomic lineage information directly, but rather allows the user to define three relational hierarchical categories, with the biological questions of the research in mind (Figure 1, Additional file 1: Figure S1). This approach allows flexibility, because it can be scaled to the level of taxonomic/phylogenetic interest (e.g. intraspecies, intragroup, etc.), and it can be adjusted to frequent 


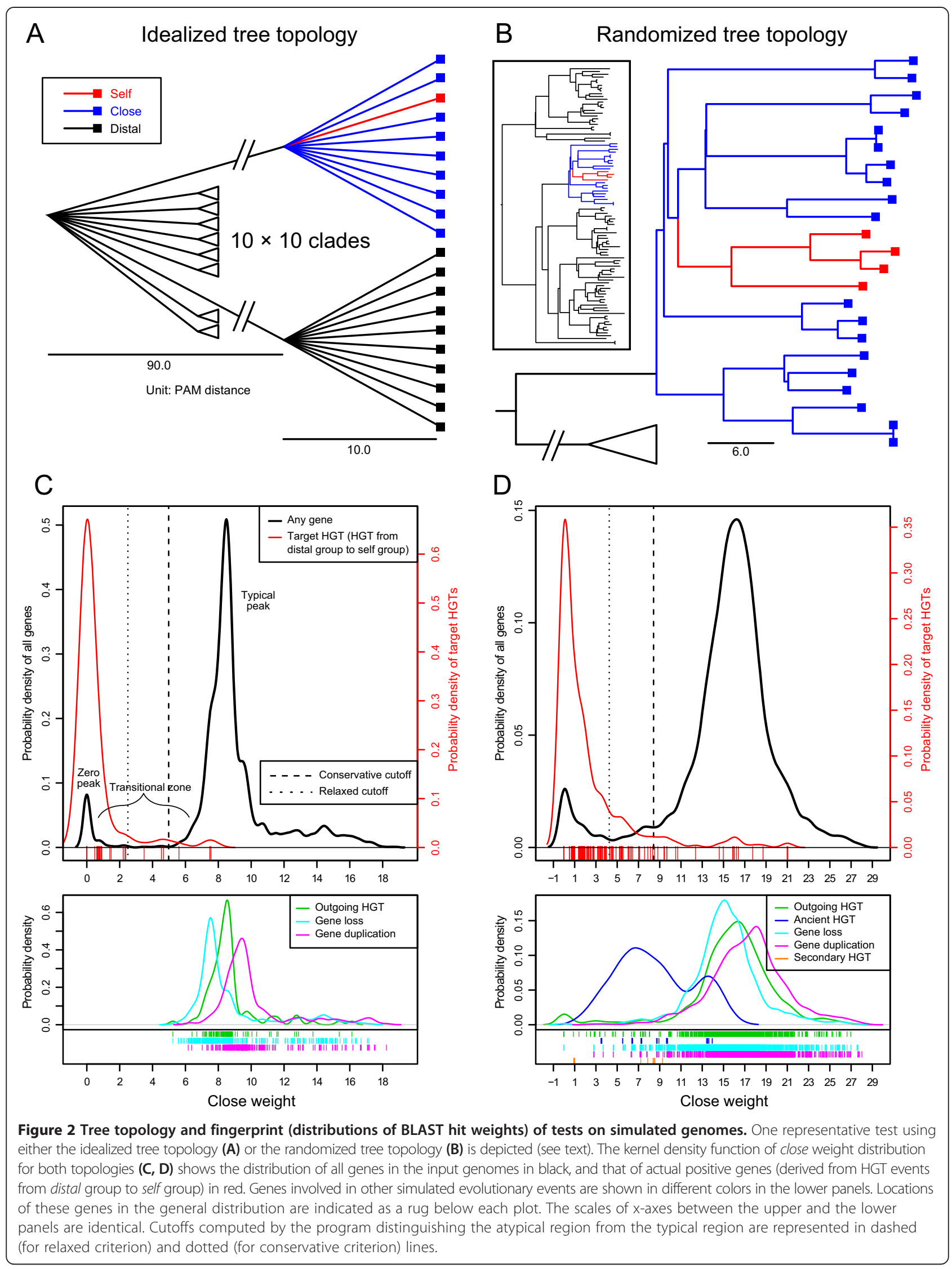


updates in taxonomy. The self group is considered the recipient, and always has to include the query genome (s), and, depending on analytical scale may also include its immediate sister organisms (e.g., different strains within the same species, or different species within the same genus). The close group will include representatives of the putative vertical inheritance history of the group (e.g., other species of the same genus, or other genera of the same family which the query genome belongs to). The distal group includes all other organisms, which are considered phylogenetically distant from the query genome (e.g., other families, orders, etc.). The method will then aim to identify genes that are likely derived from directional gene flow from groups of organisms within the distal group to members of the self group.

We introduced a measure to quantify quality of BLAST hits of each group. This measure ("weight") is calculated per group by summing up the normalized BLAST bit scores of hits. Because three categories were defined a priori, this step will result in three weights per gene. The three weights of all genes of the query genome are considered as three independent statistical populations. If multiple genomes are analyzed together, the weight populations can be merged. The three distributions together are defined as a fingerprint of the input genome(s).

A cutoff is set up to divide the weight distribution of each group (self, close and distal) into typical (larger than or equal to cutoff value) and atypical (smaller than cutoff value). If most genes of the genome(s) have a vertical inheritance history, the typical portion should include a majority of the genes, while the atypical portion should only include a small, but significant subset of genes whose hits of this specific group are underrepresented. The cutoff defines the stringency of prediction: the higher the weight cutoff, the more genes are considered as being atypical. The pipeline implements statistical approaches to compute the cutoffs, but the user is free to implement and use their own statistics. Because each genome or set of genomes generates a different fingerprint, cutoffs will vary, and are not transferrable across analyses.

For any gene to be predicted as putatively horizontally acquired, the following rules apply, which take into account all three weight distributions for that gene:

Rule 1 The gene is below cutoff (atypical) in the close weight distribution, suggesting that the orthologs of the query gene are absent in all or most of the sister groups of the organism of interest. This means that the BLAST hits belonging to this group are significantly underrepresented, in terms of number of hits or bit score, or both (Additional file 1: Figure S1B-D).
This phenomenon can be explained by: (i) the gene lacks a vertical inheritance history, or alternatively, (ii) the gene was vertically inherited, but underwent multiple independent gene loss events in the sister groups, a case that is usually less likely to be true [11]. In the ideal scenario of a high likelihood of HGT, the weight should be zero, meaning that there is no close hit (Additional file 1: Figure S1B,C). In real datasets however, there are sometimes sporadic close hits with low bit scores (Additional file 1: Figure S1D). These hits may be due to stochastic sequence similarity, secondary HGTs, paralogous genes or other mechanisms. This situation typically causes false negatives in conventional best BLAST match methods [11]. However, in the present method, these sporadic hits will not significantly alter the overall weight of a gene, thus hardly affecting the prediction results.

On the other hand, for a vertically transmitted gene, its orthologs may not always be present in each and every sister lineage. Occasional gene loss events may take away some of the expected number of close hits (Additional file 1: Figure $\mathrm{S} 1 \mathrm{E})$. This situation is a major source of false positives in best BLAST match methods [31]. However, this problem is effectively overcome in the present method, because sporadic absences of hits do not make the overall weight atypical. Moreover, one or a few high-score distal hits caused by natural (outgoing HGT from the self group to the distal group) or artificial reasons (contamination, mislabeling, etc.) (Additional file 1: Figure S1F) can easily deceive conventional methods [32]. However, the present method is immune to this problem, as the close weight remains unchanged in this situation.

Rule 2 The gene is equal to or above cutoff (typical) in the distal weight distribution, meaning that the hits from distant organisms are not underrepresented (Additional file 1: Figure S1A-F,H). This criterion sets up a filter at the donor side of potential HGT events: given the gene was transferred from a representative or ancestor of organisms that belong to the distal group, BLAST hits of the distal group itself should not be underrepresented. Otherwise, it is not convincing to conclude that the gene is HGT-derived.

The goal of this rule is stringency, in order to better distinguish putative HGT events from other scenarios that can also make a gene's close weight atypical: (1) de novo gene origination within the self group, (2) inaccurate genome annotation that considers a non-coding region a 
gene, or (3) HGT from an unsequenced organism that is not phylogenetically close to any sequenced groups of organism. These scenarios usually result in few to no distal hits (Additional file 1: Figure S1G, upper panel). Meanwhile, (4) sequence similarity due to randomness instead of homology may also bring in some distal hits with low bit scores (Additional file 1: Figure S1G, lower panel).

Rule 3 (optional) The gene is below cutoff (atypical) in the self weight distribution. It means that a gene is only sporadically detected, rather than being prevalent across the whole self group of organisms (Additional file 1: Figure S1B, H). This option will restrict the prediction to a subset of putatively HGT-derived genes that were acquired by specific lineages of the self group, instead of the whole self group. Often, these could be recent transfer events.

To assess the source of a putative HGT event, the best match organism from the distal group is reported. It is important to understand that this best match is likely not the actual physical donor, but may be an extant representative of an ancestral, extinct donor. We recommend using "donor link" to describe the directionality of transfer, and relationship between these organisms, instead of "donor" [37].

A flowchart of the procedures of this method is illustrated in Figure 1.

\section{Computational pipeline General procedure}

The HGTector pipeline (publically available at: https:// github.com/DittmarLab/HGTector) is written in Perl, and is cross-platform supported, running in Windows, Mac OS and Linux systems. It requires only the Perl interpreter with its core modules, a default component of most Mac OS and Linux distributions and is very easy to install in Windows systems. In the default mode, the program depends on no additional software or local databases to run. This characteristic maximizes the ease of installation for users without professional computer background and resources. Optionally, it calls $\mathrm{R}$ [42] to perform advanced statistical computing and graphing. Parameters of the program are managed by a central configuration file, which can be created and edited manually or via a graphical user interface (GUI). The program is composed of the following procedures:

First, the program performs batch BLASTP of protein products of multiple genes supplied by the user. Multiple formats ranging from simple lists of NCBI accession numbers to annotated genomes in GenBank format are supported. It runs BLASTP either via web connection to the NCBI server, or with a standalone BLAST program and a local database. Faster alternatives to the original BLASTP may also be used, as long as their output files are in a compatible format. It also harvests taxonomic information of each hit for each gene from the NCBI taxonomy database. Hierarchical taxonomic reports (NCBI TaxBlast) and sequences of hits (original or aligned) can be retrieved optionally. BLAST results are saved in NEXUS format [43], which can be directly viewed by text editors, or opened as multiple sequence alignments by external programs such as SeaView [44], for additional analyses. This characteristic facilitates downstream analyses, and compatibility with other programs.

BLAST hits are filtered by multiple optional functions to overcome putative taxon sampling bias that may affect BLAST hit distribution: (1) When multiple hits are present for one organism (e.g., dozens of copies of a mobile element), only the best hit is maintained, representing the putative ortholog of the query protein [45]. (2) When multiple genomes are present for one species (e.g., hundreds of sequenced Escherichia coli strains), only the genome that contains the best hit is maintained. (3) Taxonomic name keywords or IDs that represent unwanted BLAST targets, such as inaccurately defined taxonomic ranks (e.g., genus Clostridium) and biological categories (e.g., "environmental samples"), can be specified so that these hits will be omitted.

By default, the program will exclude genes without any non-self hits from subsequent analyses, because they may represent ORFans [46], resulting from de novo gene origination events (which are very rare [47]), or transfer events from unknown sources that are very dissimilar from any sequenced genomes. Alternatively, they may represent genome annotation errors, which have been long recognized as a common and perturbing issue [48-50]. While these genes are not considered in the subsequent analysis, the genes are reported as "putative ORFans or annotation errors", or POE, in this pipeline (Additional file 1: Figure S1G, upper panel). This allows the user to check which POEs were omitted, and if necessary make further adjustments to the analytical set up.

Based on the retrieved taxonomic information, the program can automatically formulate a grouping scenario, in which the lowest rank that includes all input genomes is defined as the self group, and the next higher rank as the close group. Because taxonomic classifications in GenBank may not always reflect the most current natural groupings of organisms, users may manually define hierarchies based on current knowledge of phylogeny and the purpose of their research.

With a properly defined grouping scenario, the program then calculates the three weights of each gene, computes a fingerprint of the whole genome(s), defines proper cutoffs and determines the population of atypical 
events, and possible HGTs based on the selected rules. Basic statistical parameters of the three distributions of weights, as well as the weight populations themselves are reported. The fingerprint may be visualized by box plots, histograms, density plots and scatter plots (Figures 2 and 3). Statistical analyses and graphing of BLAST hit distributions are automated in the program. They are performed using Perl codes, or by sending commands to R [42]. The communication between Perl and R is utilized by the Perl module "Statistics::R". While multiple statistical approaches are available for the user's choice, the typical procedures, which are used in the tests described in this article, are as follows:

\section{Cutoff determination}

The program performs kernel density estimation [51] to obtain a function of probability density distribution of the close weight for all genes. By default it uses Gaussian

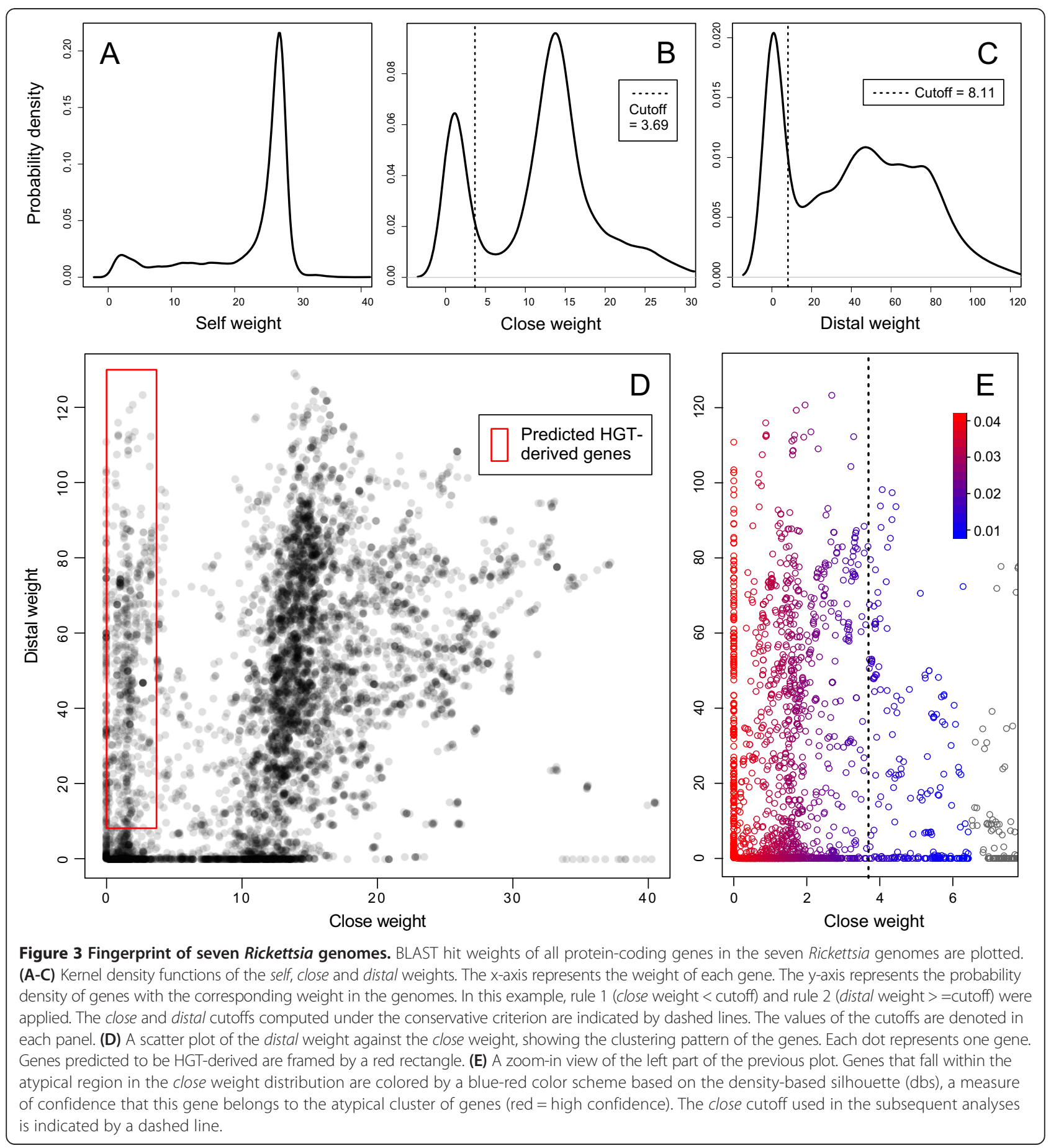


kernel smoothing with Silverman's rule-of-thumb bandwidth selector [52]. The user is allowed to choose a proper bandwidth selection factor that controls the smoothness of the curve. The function is plotted and made visible to the user in real time. Statistically significant local minima (pits) and maxima (peaks) are computed using the "pastecs" package [53] with default parameters following Kendall's information theory [54], and their $\mathrm{x}$-coordinates are recorded and displayed to the user. The program then automatically identifies a local minimum separating the typical from the atypical proportion of the gene population under consideration. Specifically, the biggest peak or group of continuous peaks in terms of number of genes it covers is identified as the typical region, and the rest is defined as the atypical region (Figures 2 and 3).

In addition to the apparent typical peak, there is usually a clearly identifiable peak located close to zero (referred to as the "zero peak" hereafter). This peak usually includes "ideal" putatively HGT-derived genes (=zero BLAST hit). Between the zero peak and the typical peak is a transitional zone that likely consists of genes with ambiguous evolutionary history (see Results and Discussion).

The program automatically reports two cutoffs: the $\mathrm{x}$-coordinate of the identified local minimum is naturally chosen as a cutoff (referred to as the "relaxed cutoff" hereafter). However, based on results of repeated tests, we recommend using the second cutoff, which is defined as the arithmetic mean of the $x$-coordinates of the local maximum of the zero peak and the local minimum of the selected pit (referred to as the "conservative cutoff" hereafter) (Figure 2C, D). The choice between the two cutoffs depends on the goal of research, but for the identification of putative HGT events, the conservative cutoff is preferred as it meets a balance between precision and recall (see Results and Discussion).

The program also implements several functions to assess the statistical significance of separating atypical genes from typical ones. For the whole weight population, the program performs Hartigans' dip test [55] to assess the nonunimodality of the weight distribution, which essentially is the statistical significance that a distribution can be divided into two or more distinct parts. The test is performed by calling the "diptest" package in $\mathrm{R}$ [56]. The dip statistic and the $\mathrm{p}$-value for the test for unimodality are reported. For each individual gene, the program computes a density-based silhouette (dbs) (using the "pdfCluster" package in R), which is a statistical measure of confidence that a certain data point (gene) is allocated to a cluster (here the atypical region) [57].

The same procedures apply to the distribution of the distal weight (and the self weight, if the optional rule 3 is chosen). In addition to the above described statistical approaches, power users may also perform extra statistical analyses based on the weight data output by the program, and type user-defined cutoffs.

Based on the cutoffs, the program reports a population of genes with an atypical, non-vertical history, which in the context of the a priori provided phylogenetic information represents a putative horizontal history. The results are summarized in a choice of plain text, web page (HTML) or Excel spreadsheet formats. The latter allows for convenient downstream statistics of outputs, and includes hyperlinks that allow users to track each of the genes back to their original BLAST report. It not only reports the number and percentage of putatively HGT-derived genes, but also optionally categorizes each gene in three contexts: (i) By putative donor group, which is described by userdesignated higher taxonomic rank of the best match organism (based on GenBank annotations). (ii) By functional annotation of protein products, which is provided by external sources, such as the output of Blast2GO [58]. (iii) By gene orthology (evolutionary history of each individual gene family across input genomes), which is identified by a built-in function of BLAST hits clustering or from external sources, such as the output of OrthoMCL [59]. These reports (for example see Additional file 1: Figure S1G) allow users an intuitive view of the prevalence of HGT-derived genes and the evolutionary, ecological and functional implications of HGTs at levels of individual genes, gene families, whole genomes and multiple phylogenetically related genomes.

The whole analysis can be performed on a regular personal computer, as it does not require extensive computing power. Batch-BLAST is the most time-consuming step, which typically lasts several hours to several days, depending on the number of protein-coding genes in the genome(s) of interest. The computation time can be reduced if faster BLAST alternatives or smaller databases are used. The statistical analysis typically takes only minutes. Data generated by the program can be parsed and re-used by other programs for multiple purposes.

As an additional, and important function, the program provides a complete phylogenetic pipeline, which automates the process of multiple sequence alignment, alignment trimming and phylogenetic tree reconstruction, by calling external local programs such as ClustalW [60], Gblocks [61] and RAxML [62], and parsing their outputs. Reconstructed phylogenetic trees are annotated with organismal names and are attached to BLAST reports, which in turn can be directly viewed by external programs such as FigTree [63]. This function allows users to monitor and validate prediction results by manually checking the evolutionary scenarios of individual genes.

\section{Analysis of simulated genomic data}

To assess the performance of this method under the impact of various evolutionary scenarios, as well as to compare 
HGTector to conventional BLAST methods, we tested the above-described pipeline on simulated genomic data.

\section{Simulation of genome evolution}

Simulated genomic data were generated by ALF (Artificial Life Framework) version 1.0, a program that simulates genome evolution [64]. In each simulation, 100 species evolved from one randomly generated root genome containing 1000 protein-coding genes that are no shorter than 50 aa. During the process random inter-genomic HGT events occurred under a pre-defined global rate, which varied between simulations (see below). The process also simulated the following evolutionary forces in addition to HGT, at random rates: speciation, character substitution, insertion and deletion, GC-content amelioration, rate variation among sites and among genes, gene duplication and loss. Many of these forces are known to affect HGT prediction $[15,31,32]$.

Two types of simulations were performed. First, an idealized, pre-defined tree topology was used, in which all representatives of close- and self-designation are grouped in a polytomy, signifying (in this case) equal genetic distance, thus eliminating the impact from evolutionary artifacts. The rest of the taxa are placed relatively distant (unrelated) in the tree to lower the possibility of stochastic BLAST matches. The topology is detailed as follows: 10 clades branch from the tree base simultaneously at time point 0 (unit: PAM distance, same below). In each clade, 10 species branch off simultaneously at time point 90 . The tips are at time point 100 for all species. For each clade, one species was randomly chosen as the input genome (also the self group), while nine species were defined as the close group. All 90 species in other clades are considered as the distal group (Figure 2A). All ten clades were analyzed in this manner. This simulation was replicated 10 times (=100 analyses), with the HGT rate ranging from 0 (negative control) to 0.0045 with an interval of 0.0005 .

Second, a randomized birth-death tree was generated in ALF per simulation (birth rate $=0.1$, death rate $=0.01$, height $=1000)$, mimicking a more realistic topological situation. A random clade was manually chosen from the tree, as long as it met the following criteria: (1) 3-8 self species that formed a clear monophyletic group; (2) 10-20 close species that were closely related to the self clade; (3) the self and close species together formed a clear monophyletic group that was independent from all other species (the distal group) (Figure 2B). This simulation was replicated for 100 times, with the HGT rate of each replicate randomly sampled from a range of 0 to 0.005 .

Evolutionary events (HGT, gene duplication, gene loss, etc.) simulated in each analysis were extracted from the ALF log file. The time, species and genes involved in these events were recorded. HGT events involving the self group were further categorized by their donor, recipient and time into the following groups: target HGT (HGT from the distal group to the self group, which are the actual positives to be targeted by HGTector), ancient HGT (HGT from the distal group to the ancestor of the self group), outgoing HGT (HGT from the self group to the distal group, which is equivalent in consequence to a database error that mislabels a sequence with another organism), and secondary HGT (target HGT combined with one or more additional transfers between the self and the close groups). Gene loss and gene duplication events taking place in the close group were singled out as they directly influence the distribution of the close weight.

For each simulation (idealized or randomized tree topologies), a BLASTP database including the protein sequences from all 100 genomes was created using the standalone BLAST program [17]. All-against-all BLASTP was performed with an E-value cutoff at $1 \times 10^{-5}$ for all genes in the selected self genomes, considering at least 20 hits. In order to demonstrate the effect of gene duplication on the prediction result, the program option of excluding paralogs was turned off.

A modified version of the HGTector pipeline was created to parse the ALF outputs and to perform analysis. Both the conservative and the relaxed cutoff criteria were tested. The fingerprint was plotted along with the distribution of actual positives (target HGTs) as well as other evolutionary scenarios (see above) for manual observation in addition to statistical analysis.

\section{Comparison to conventional BLAST approach}

The performance of HGTector in the context of identifying atypical and putatively HGT-derived genes, was compared against that of commonly used conventional BLAST-based methods by modifying the pipeline to mimic the conventional approach, which does not consider overall hit distribution. Specifically, we considered HGT-events for the scenario where no close hits are present (criterion: $\mathrm{C}=0$ ); or the scenario where there is at least one distal hit with a bit score higher than those of any close hits (criterion: $\mathrm{D}>\mathrm{C}$ ).

\section{Assessing performance}

The predicting power in the context of target HGTs under each criterion, as well as for conventional BLAST was assessed by precision and recall. Precision describes the number of true positives over the number of all predicted positives (=How many of the predicted HGTs are real), and recall describes the number of true positives over number of all actual positives (=How many of all real HGTs were found), which are commonly used performance markers for binary classifications. Statistical analysis and plotting were conducted using $\mathrm{R}$ [42]. 


\section{Application of HGTector to real datasets}

HGTector was further tested on a variety of real-world genomic data, covering bacteria (5), unicellular eukaryotes (1) and human (1) (Table 1), the last of which serves as a realistic negative control since HGTs into genomes of higher animals are known to be very rare [5]. The most in depth analysis was conducted on the Rickettsia dataset, because it affords comparisons to previous results obtained with a variety of methods $[29,36,37,39,65,66]$.

\section{Analysis on the Rickettsia dataset}

Out of all available Rickettsia genomes, we selected seven representative Rickettsia species with fully-annotated genomes for this analysis (Table 1). All of these species belong to the spotted fever group (SFG), a traditional classification group of Rickettsia. A grouping scenario was chosen based on the taxonomy and phylogeny of major Rickettsia species, which has been well resolved by recent studies [37,41,67]. Specifically, we defined the self group as SFG (NCBI taxonomy ID: 114277), the close group as order Rickettsiales (766), excluding SFG, and the distal group as all nonRickettsiales organisms.

All-against-all BLASTP was performed against the NCBI non-redundant protein sequences (nr) database with an Evalue cutoff at $1 \times 10^{-5}$. A soft filtering for low sequence complexity regions, which was suggested as the optimal parameter for ortholog identification [68], was used. Hits with organism names containing these words were excluded for the purpose of this analysis: unknown, uncultured, unidentified, unclassified, environmental, plasmid, vector, synthetic, and phage. Up to one hit from each organism was preserved. A maximum number of 200 hits were preserved for each protein. A global fingerprint was computed and graphed to describe the pattern of BLAST hit distribution of all seven genomes. Cutoffs for the three groups of weights were computed using the built-in kernel density estimation function under the conservative criterion. The default rules 1 and 2 were applied.

\section{Assessing stochastic events using real datasets}

To test the impact of stochastic events on prediction results, the following simulations were carried out on the Rickettsia dataset: (1) database error (some sequences are mislabeled by incorrect organism names), (2) gene loss in the close group, (3) rate variation in the close group, (4) rate variation in the input genomes, (5) taxon sampling bias (some groups of organisms are overrepresented in the genome database), and (6) smaller sample size of genes. Multiple degrees of modification intensity $(x)$ for each type of events were tested, each having 100 replicates (except for taxon sampling bias). Specifically:

1. Database error. For each hit, there is $x$ proportion of chance that its corresponding organism was assigned to an organism randomly sampled from the pool of BLAST results of all genes.

2. Gene loss in the close group. For each close hit, there is $x$ proportion of chance of being removed from the BLAST hit table.

3. Rate variation in the close group. For each close hit, there is $x$ proportion of chance that its bit score is divided by a factor subject to a gamma distribution with shape parameter $k=2$ and scale parameter $\theta=1$ :

$S_{1}=S_{0} /(1+\Gamma(2,1))$

Where $S_{0}$ and $S_{1}$ refer to the bit score before and after manipulation, respectively.

4. Rate variation in the input genomes. For each query gene, there is $x$ proportion of chance that the bit scores of all its hits are divided by a factor subject to a gamma distribution same as above.

5. Taxon sampling bias. For selected representative groups of organisms from close and distal groups (see Results and Discussion), all hits belonging to this group were replicated into $x$ copies ( $x$ identical but separate hits).

6. Smaller sample size of genes. $x$ out of all 8484 genes were randomly selected for fingerprint calculation in each replicate. The resulting cutoffs were in turn used for prediction on all genes. Replicates that failed to pass the Hartigans' dip test (that is, the population of either close or distal weights is not significantly non-unimodal) (p-value threshold $=0.05$ ) were counted and excluded from the analysis.

HGTector analysis was conducted on these replicates using the same procedures as in the standard analysis on the unmodified dataset (see above). The results were compared to the results derived from a conventional BLAST-based approach under the $\mathrm{D}>\mathrm{C}$ criterion (see Analysis of simulated genomic data). Precision and recall of the results were computed using the result of the standard analysis as the reference.

\section{Cross-method comparison}

In order to evaluate the performance of HGTector on the Rickettsia dataset in the context of other available methods, results were compared to two examples from each of the three currently employed strategies: BBH (bidirectional best hit) [19] and DarkHorse [28,29] based on best BLAST match; GIST [69] and IslandViewer [66] based on sequence composition, and two studies conducted by Merhej et al. [37] and Le et al. [36], using phylogenetic approaches. We exemplified this comparison on the $R$. felis genome, which previously has been demonstrated to have high HGT frequency $[37,39,65]$. 
Table 1 Real genomic datasets tested in this study

\begin{tabular}{|c|c|c|c|c|c|c|}
\hline Category & Self group & Close group & No. of genes & Date of BLAST & Max. no. of hits & $\begin{array}{l}\text { List of input genomes (organism name and } \\
\text { NCBI accn. no.) }\end{array}$ \\
\hline \multirow[t]{7}{*}{ Alphaproteobacteria } & SFG Rickettsia & Rickettsiales & 8484 & Jan. 2013 & 200 & R. akari str. Hartford [GenBank:NC_009881] \\
\hline & & & & & & R. felis URRWXCal2 [GenBank:NC_007109] \\
\hline & & & & & & R. massiliae MTU5 [GenBank:NC_009900] \\
\hline & & & & & & R. slovaca 13-B [GenBank:CP002428] \\
\hline & & & & & & R. rickettsii str. 'Sheila Smith' [GenBank:NC_009882] \\
\hline & & & & & & R. africae ESF-5 [GenBank:NC_012633] \\
\hline & & & & & & R. conorii str. Malish 7 [GenBank:NC_003103] $]^{1}$ \\
\hline \multirow[t]{6}{*}{ Firmicutes } & Streptococcus & Bacilli & 11906 & Nov. 2013 & 100 & S. anginosus C238 [GenBank:NC_022239] \\
\hline & & & & & & S. gallolyticus UCN34 [GenBank:NC_013798] \\
\hline & & & & & & S. intermedius B196 [GenBank:NC_022246] \\
\hline & & & & & & S. mutans LJ23 [GenBank:NC_017768] \\
\hline & & & & & & S. pneumonia A026 [GenBank:NC_022655] \\
\hline & & & & & & S. suis JS14 [GenBank:NC_017618] \\
\hline \multirow[t]{6}{*}{ Epsilonproteobacteria } & Helicobacter & Campylobacterales & 10531 & Mar. 2013, Nov. $2013^{2}$ & 200 & H. acinonychis Sheeba [GenBank:NC_008229] \\
\hline & & & & & & H. bizzozeronii CIII-1 [GenBank:NC_015674] \\
\hline & & & & & & H. cinaedi PAGU611 [GenBank:NC_017761] \\
\hline & & & & & & H. felis ATCC 49179 [GenBank:NC_014810] \\
\hline & & & & & & H. mustelae 12198 [GenBank:NC_013949] \\
\hline & & & & & & H. hepaticus ATCC 51449 [GenBank:NC_004917] \\
\hline \multirow[t]{5}{*}{ Gammaproteobacteria } & Erwinia & Enterobacteriales & 19013 & Mar. 2013 & 200 & E. amylovora ATCC 49946 [GenBank:NC_013971] \\
\hline & & & & & & E. billingiae Eb661 [GenBank:NC_014306] \\
\hline & & & & & & E. sp. Ejp617 [GenBank:NC_017445] \\
\hline & & & & & & E. pyrifoliae DSM 12163 [GenBank:NC_017390] \\
\hline & & & & & & E. tasmaniensis Et1/99 [GenBank:NC_010694] \\
\hline Actinobacteria & Mycobacterium africanum & Mycobacterium & 3830 & Oct. 2013 & 100 & M. africanum GM041182 [GenBank:NC_015758] \\
\hline Unicellular red algae & Galdieria sulphuraria & Eukaryota & 7174 & Dec. 2013 & 50 & G. sulphuraria [GenBank:ASM34128v1] ${ }^{3}$ \\
\hline Higher animal & Homo sapiens & Animalia & $22516^{4}$ & Nov. 2013 & 1000 & H. sapiens [GenBank:GCF_000001405.13] \\
\hline
\end{tabular}

${ }^{1}$ The genomes used in this study are identical to those used in [37].

${ }^{2}$ Two independent analyses were conducted on different dates, and similar outcomes were obtained. The more recent result was reported.
${ }^{3}$ The genome used in this study is identical to that used in [79].

${ }^{4}$ For genes with multiple isoforms, the longest CDS was extracted using an in-house Perl script and used for the analysis. 
Specifically, BBH analysis was performed as a built-in function of the present pipeline. This method is similar to the $\mathrm{D}>\mathrm{C}$ criterion as described above, except for an additional reverse BLAST step with the same parameters to confirm that the two sequences are each other's best match within their host genomes. The result by DarkHorse was downloaded from the DarkHorse server (darkhorse.ucsd.edu). Default parameters were used, in which the BLASTP E-value cutoff is also $1 \times 10^{-5}$. All three available phylogenetic granularities, strain, species and genus, were used and the results were merged, in order to maximize the discovery rate. Both GIST and IslandViewer are targeting large pieces of heterogeneous genomic regions, or genomic islands (GI) [70]. GIST is a synchronization of five subprograms: AlienHunter [71], IslandPath [72], SIGI-HMM [73], INDeGenIUS [74] and PAI-IDA [75]. The subprograms were executed in a local system and the results were processed using the EGID algorithm [76] to get a consensus result. IslandViewer is an integration of three subprograms: IslandPick, SIGI-HMM and IslandPath. The integrated result was downloaded from the IslandViewer server (www.pathogenomics.sfu.ca/ islandviewer). Results of GIST and IslandViewer were further processed by an in-house Perl script to extract the genes included in the genomic islands. The putative HGT-derived genes predicted by Merhej et al. [37] and by Le et al. [36] were extracted from the original publications. Specifically, Merhej et al. identified 152 HGT-derived genes in the $R$. felis genome that are linked with organisms other than SFG Rickettsia [37]. Le et al. identified 11 instances of HGT from outside Rickettsiales into the R. felis genome [36].

The predicted HGT-derived genes or genomic islands by different methods were spatially mapped to the $R$. felis genome and visualized in Geneious 6.0 [77]. An "overlap factor" (OF) was employed as a criterion to compare the outcomes of different methods by assessing the overlap. This was expressed as the negative logarithm of the likelihood that the overlap was obtained by chance. To compute an OF, the number of the same genes predicted by each method pair was counted, and the OF was calculated following the probability mass function of the hypergeometric distribution:

$$
O F=-\log \left(\left(\begin{array}{l}
a \\
k
\end{array}\right)\left(\begin{array}{l}
n-a \\
b-k
\end{array}\right) /\left(\begin{array}{l}
n \\
b
\end{array}\right)\right)
$$

Where $n$ is the total number of genes; $a$ and $b$ are the numbers of genes predicted by two methods, respectively; $k$ is the number of genes overlapping by two sets of results. The larger an OF is, the more overlapping, and thus more consistent the two sets of results are, and the more likely it is that the two methods are identifying the same group of genes.

\section{Results and discussion}

\section{Performance on simulated genomic data}

\section{Testing precision and recall}

In all experimental groups under the idealized tree topology, a clear bimodal distribution was observed (Figure 2C), which is expected when HGT is present in the data. Meanwhile, none of the negative control groups have an identifiable zero peak, which is equivalent to a vertical history for all genes (no HGT events). Both cutoff criteria achieve high precision and recall simultaneously. In particular, under the conservative criterion, $99.4 \%$ of the prediction results are true positives. Meanwhile, they cover over $91.3 \%$ of all actual HGT-derived genes. The more relaxed criterion still achieves a precision of $95.3 \%$ and a recall of $96.8 \%$.

In tests under randomized tree topologies, a larger transitional zone is present between peaks of the expected bimodal distribution, which is also frequently observed in real datasets. Both, precision and recall are affected compared to the idealized scenario, but in different measures. The conservative cutoff still maintains reasonably high precision (81.6\%) and recall (90.5\%) simultaneously, relative to the known number of HGT events. The relaxed criterion keeps equally high recall $(96.6 \%)$ but its precision drops significantly (42.6\%) (Figure 4, Additional file 1: Figure S2).

Because the test under randomized topologies is a more realistic representation of datasets, its result serve as a better reference for the practical consideration of the HGTector application. Given the simultaneously high precision and recall, we recommend using the conservative cutoff for both initial HGT candidate screening (to be followed by phylogenetic analysis or other analyses) and standalone HGT discovery (when further in-depth analyses are not applicable). The relaxed cutoff may be considered only when the user wants to maximize discovery rate in an initial screening, in spite of its higher false positive rate. The comparison between idealized and randomized topologies further indicates the positive correlation between prediction success and a properly defined grouping scenario, in which: (1) the self-close clade is relatively distant from any other organisms; and (2) there are multiple subclades in the close group, each having similar number of taxa represented in the database.

Varying global HGT rates seemingly show little effect on the stability of precision and recall of this method (Additional file 1: Figure S3), suggesting that the predicting power is independent of HGT rate.

In comparison, the performance of both conventional BLAST-based approaches $(\mathrm{C}=0$ and $\mathrm{D}>\mathrm{C})$ is notably unbalanced. Atypical genes falling under the $\mathrm{C}=0$ criterion have the highest precision $(100.0 \%$ and $97.1 \%$, for idealized and randomized tree topology, respectively), but very low recall $(78.1 \%$ and $73.7 \%)$. The D > C 


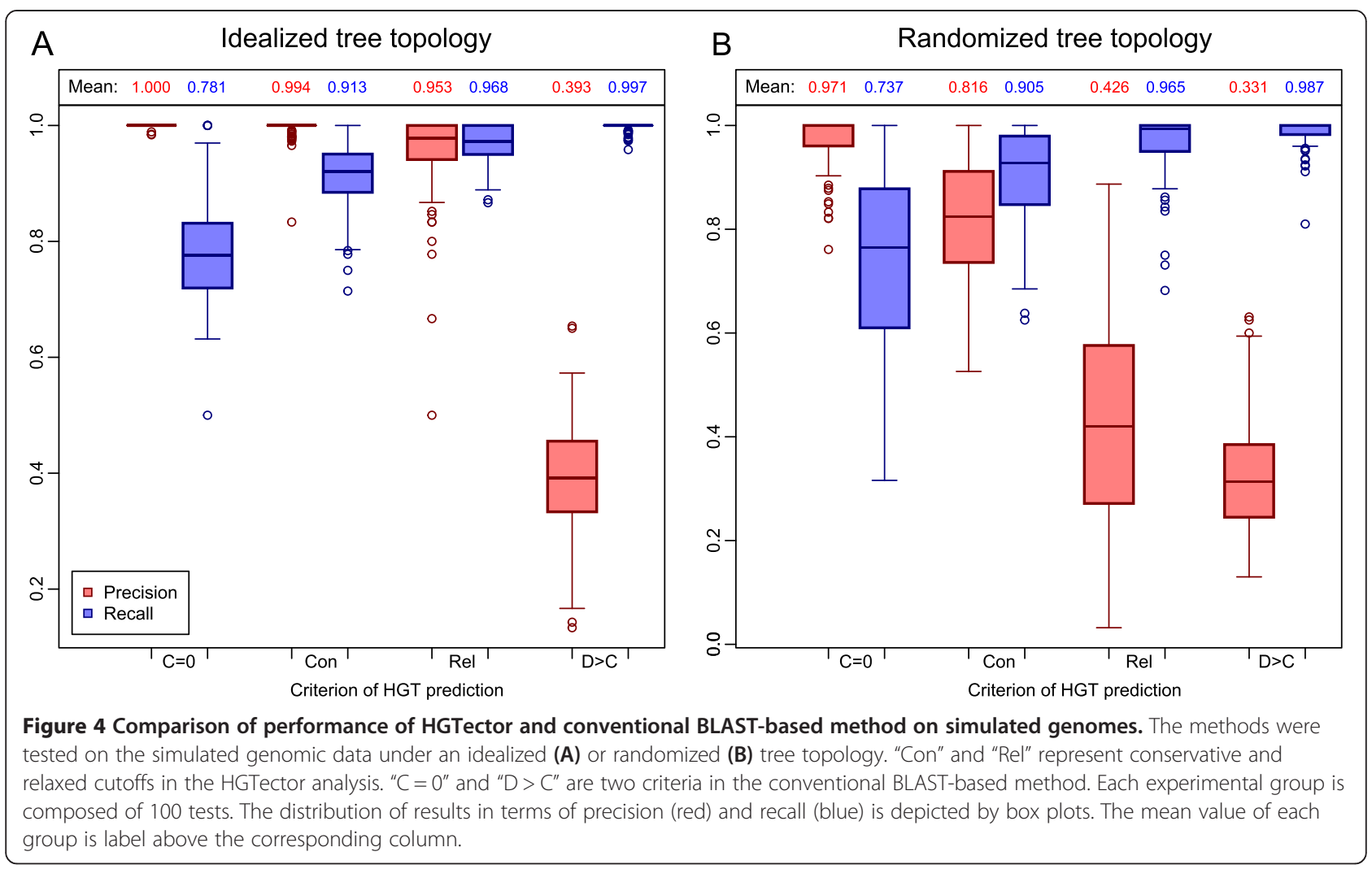

criterion has high recall $(99.7 \%$ and $98.7 \%)$ but extremely low precision (39.3\% and $33.1 \%$ ), showing an intolerably high false positive rate (Figure 4, Additional file 1: Figure S2). From a practical perspective, $\mathrm{C}=0$ is too stringent, thus omitting a big portion of true HGTderived genes affected by stochastic events, while $\mathrm{D}>\mathrm{C}$ is too relaxed and not capable of differentiating genes that have high-score distal hits merely due to stochastic reasons instead of HGT (see below). It should be mentioned that $\mathrm{C}=0$ is not applicable to real datasets due to frequent genome annotation errors and ORFans, both of which may have a zero close weight.

\section{Evaluating other evolutionary scenarios}

The impact of other evolutionary events on the fingerprint and the division between typical and atypical gene populations was explored. Outgoing HGT (from self to distal) seemingly does not significantly alter the close weight of a gene. Gene loss decreases close weight and gene duplication increases it, both within an insignificant range (Figure 2C, D). Most importantly, genes within these three categories of evolutionary history still fall within the typical region and were not mistakenly detected as atypical by HGTector.

It is particularly notable that the majority of genes derived from ancient HGT events are located in the transitional zone (Figure 2D). Expectedly, they constitute a considerable portion of false positives in our analyses. In other words, depending on cutoff, more of them are likely to be placed in the typical population, instead of the atypical, and presumably non-vertical population. A similar pattern was observed for secondary HGT events. Although not frequent in the simulated genomes here, these events are actually very frequent in real datasets, as HGT frequency is higher between closely related organisms [78]. However, in the conventional BLAST method $(\mathrm{D}>\mathrm{C})$ most false positives are composed of mainly outgoing HGTs but only a few ancient HGTs.

\section{Application to real datasets}

The close weight distributions for real datasets exhibit a bimodal distribution containing a broad typical region and a sharp atypical peak that is located close to zero (as expected) (Figure 3A-C, Additional file 1: Figure S4). Therefore, the cutoffs that divide genes with atypical BLAST hit distribution from typical ones can be set accordingly and HGT events can be predicted based on the cutoffs. As an example, fingerprint plots for Rickettsia and Galdieria exhibit an apparent overlapping pattern between the atypical peak and the HGT-derived genes identified by previous phylogenetic studies [37,79] (Additional file 1: Figures S4E and S5D). In contrast, the human genome does not have an apparent atypical peak, which is also expected (Additional file 1: Figure S4F). 


\section{Analysis of the Rickettsia genomes}

The global fingerprint describing the pattern of BLAST hit distribution of the Rickettsia analysis is illustrated in Figure 3A-C. Results from Hartigans' dip test strongly support the non-unimodality of all three weight distributions (p-values $<2.2 \times 10^{-16}$ ). The seven Rickettsia genomes contain a total number of 8484 annotated chromosomal protein-coding genes, of which 800 genes have an atypical close weight and a typical distal weight, and thus are potentially HGT-derived (Table 2, Additional file 2: Table S1). The percentage of putative HGT-derived genes in a genome ranges from $6.05 \%$ (76 genes) in $R$. akari to $18.29 \%$ ( 256 genes) in R. felis. The number of putative HGT-derived genes per genome is positively correlated to the size of the genome $\left(R^{2}=0.755\right)$, implying contribution of HGT to the relative genome expansions in the overall reductive trend of Rickettsia genome evolution, confirming previous studies [65]. It is notable that the $R$. felis genome contains significantly more putative HGT-derived genes (256) than the rest of the genomes $(90.7 \pm 20.5$, mean and standard deviation), suggesting a particular prevalence of potential HGT events in $R$. felis evolution. The comparison between the fingerprint on R. felis genome alone (Additional file 1: Figure S5A-C) and the global fingerprint (Figure 3A-C) clearly reveals that $R$. felis has a much larger atypical peak in the close weight distribution. This reinforces outcomes from Merhej et al. [37], which found that the frequency of cross-species bacterial recombination in $R$. felis had been underestimated.

To further explore the biological information behind the predicted patterns, the results have been summarized in three separate contexts: by putative donor group, by functional annotation and by gene orthology. Similar to previous in depth analyses by Merhej et al. [37], our analyses revealed frequent donor links, such as Legionellales, Enterobacteriales and Burkholderiales, and frequently transferred gene categories, such as genes encoding transposases and genes involved in phage and plasmid activity
(Additional file 1: Figures S6 and S7; Additional file 2: Table S2).

\section{Stochastic manipulation of Rickettsia data: stability of prediction}

The results of these simulations (Additional file 1: Figures S8 and S9) suggest that HGTector's performance is notably insensitive to database error. Even under an extremely high proportion (10\%) of mislabeled genes, its precision remains close to $100 \%$. As a comparison, the precision of the conventional BLAST-based approach D > C drops below 15\% (Additional file 1: Figure S8A). Gene loss and rate variation in the close group also have remarkably weaker deleterious effects on precision of HGTector than that of conventional BLAST based methods under the $\mathrm{D}>\mathrm{C}$ scenario (Additional file 1: Figure S8B, C). Rate variations in input genomes destabilize prediction results at a certain range (5-15\%), but overall, precision remains high in HGTector when rate increases, as compared to conventional BLAST (Additional file 1: Figure S8D). Taxon sampling bias has little effect when it happens to distal organisms. However, in some close organisms (such as $R$. prowazekii and $R$. bellii), this effect may severely compromise the predicting power if the bias is strong (for example, a certain taxon group has many more representatives than others) (Additional file 1: Figure S9). Smaller sample size of input genes has a minor effect on the prediction result (Additional file 1: Figure S10), suggesting that the method is still effective on incomplete genomes. It should be noted that when the sample size is extremely small (e.g., 50 genes only), the statistical power of gene clustering can be significantly impaired (green bars in Additional file 1: Figure S10).

The above results suggest that our method is generally unaffected by stochastic events, some of which are major challenges to current methods (see Background). A concern is taxon sampling bias in the close group, an issue that can be alleviated by properly defining grouping

Table 2 Summary of genes predicted to be horizontally acquired in seven Rickettsia genomes

\begin{tabular}{lllll}
\hline Abbreviation & Size of chromosome $(\mathbf{M b})$ & $\begin{array}{l}\text { Number of chromosomal } \\
\text { protein-coding genes }\end{array}$ & $\begin{array}{l}\text { Number of predicted } \\
\text { HGT-derived genes }\end{array}$ & $\begin{array}{l}\text { Percentage of predicted } \\
\text { HGT-derived genes }\end{array}$ \\
\hline R. akari & 1.23 & 1256 & 76 & $6.05 \%$ \\
R. felis & 1.49 & 1400 & 256 & $18.29 \%$ \\
R. massiliae & 1.36 & 968 & 93 & $9.61 \%$ \\
R. slovaca & 1.28 & 1114 & 72 & $6.46 \%$ \\
R. rickettsii & 1.26 & 1342 & 98 & $7.30 \%$ \\
R. africae & 1.28 & 1030 & 78 & $7.57 \%$ \\
R. conorii & 1.27 & 1374 & 127 & $9.24 \%$ \\
Mean & 1.31 & 1212 & 114.3 & $9.43 \%$ \\
\hline
\end{tabular}


scenario and masking redundant taxonomic groups using HGTector's flexible functionality (see Methods Computational pipeline).

\section{Comparison of HGTector with other methods for analysis of} the $R$. felis genome

Together, HGTector and six other methods identified 595 putative HGT-derived genes, out of all 1400 chromosomal protein-coding genes in the $R$. felis genome (42.5\%) (Table 2, Figure 5, Additional file 2: Table S3). Of these
595 genes, 82 have been uniquely identified by HGTector. There's a considerable portion of overlapping hits between each pair of results, but none of the programs produce completely identical HGT predictions. As expected, there is relatively more overlap between two methods of the same category. Meanwhile, the overlap factors (OFs) between two methods from different categories are significantly lower. However, HGTector is a notable exception because its overlap with any other method of the three categories is significantly higher than between other

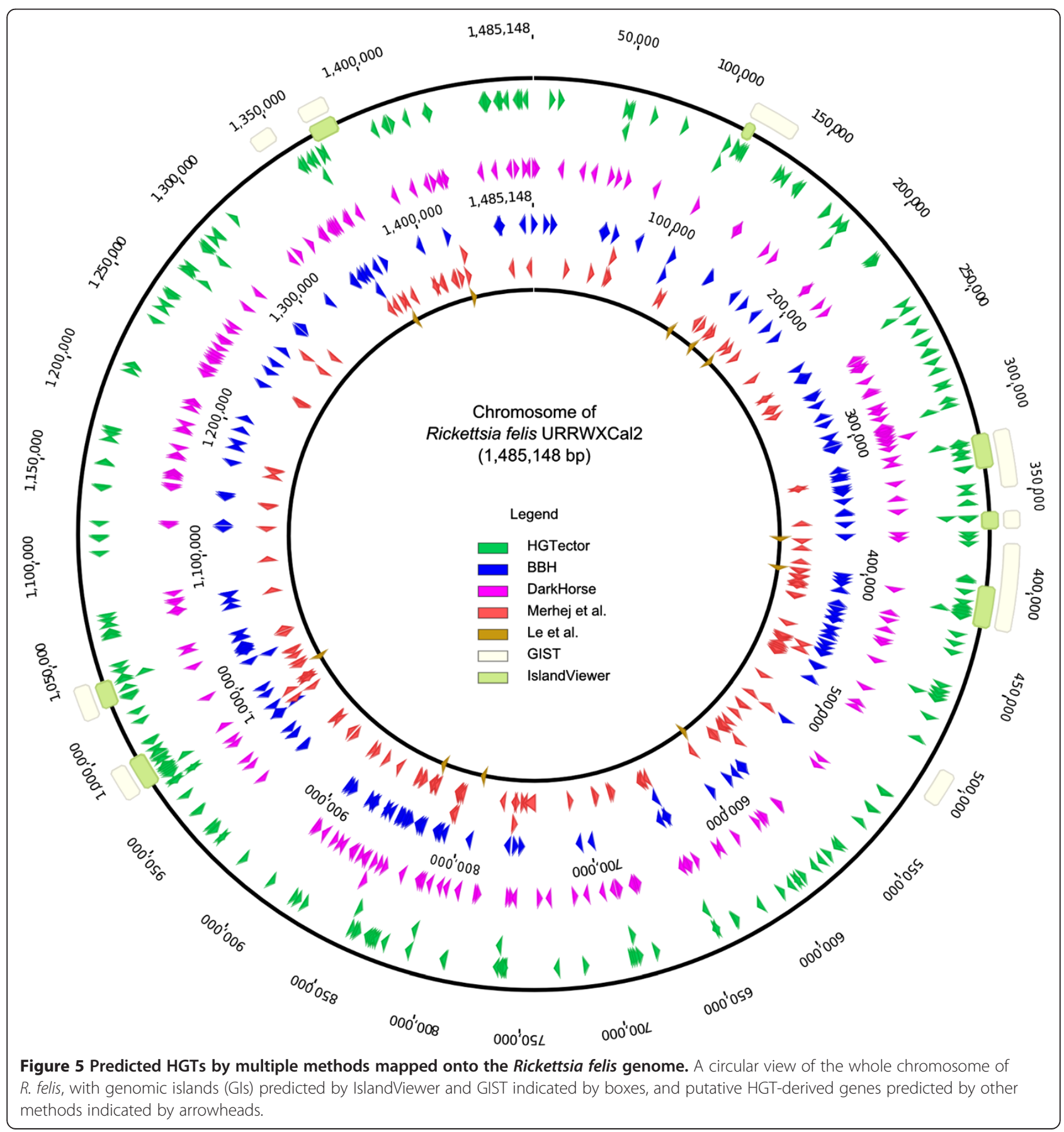


methods from different categories (Additional file 2: Table S4 and Additional file 1: Figure S11).

By comparing the results of HGTector and the results of the other two BLAST-based methods, it is noticeable that HGTector can effectively exclude false positives caused by database errors. For instance, $\mathrm{BBH}$ and DarkHorse respectively predicted 61 and 97 genes to be acquired from Ixodes scapularis (deer tick), which is a common host of Rickettsia. Therefore, it is likely that the sequenced Ixodes samples contained Rickettsia endosymbionts, and its DNA was also sequenced and indiscriminately labeled as Ixodes DNA in the unassembled shotgun sequences ([NCBI:PRJNA34667]). In other words, these are potential database errors. In contrast, in HGTector's result, none of these genes were predicted as HGT-derived, because they have considerable amount of close hits (violation of rule 1), despite the presence of a single high-score Ixodes hit.

Detection of HGT is fraught with challenges, such as ambiguity in compositional features and phyletic patterns [80], difficulty in phylogenetic reconstruction [81], interference from database error and incompleteness [49]. The observed low consistency between methods is not surprising, and has precedents in multiple previous studies [13-15]. Given the significant overlap of HGTector with all other previously employed approaches, we suggest that it is effective in producing meaningful prediction results on real datasets.

\section{Conclusions}

In this paper a novel method for genome-wide detection of vertical versus non-vertical gene history (in particular, putative HGT events) is presented. It features a statistical analysis of BLAST hit distribution patterns in the context of a priori defined phylogenetic hierarchies. The innovation of this method is the systematic consideration of all BLAST hits of all genes within selected genomes. This is in contrast to conventional BLAST-based approaches, which typically rely on a single best hit for each gene (see Background). The three-category grouping scenario is a simplified but effective implementation of prior phylogenetic knowledge into a BLAST hit distribution analysis. The set-up allows high flexibility in group assignments that best match the taxonomic level of the user's interest, as well as immediate response to frequent changes in microbial taxonomy. The advantage of this systematic approach is that it captures the overall image of gene evolution while being significantly insensitive to stochastic events. As demonstrated in this study, stochastic events such as gene loss, rate variation and database error may impose serious problem to conventional methods (see Background), but have comparatively negligible effects on HGTector.

The core assumption of the method is that the typical and atypical parts of the BLAST hit distribution are distinguishable. This assumption is repeatedly supported by tests on both simulated and real-world genomic data (Figures 2 and 3, Additional file 1: Figure S4). With the proposed procedures of computing cutoffs and the rules of targeting genes that are likely to be HGT-derived, remarkable prediction success was achieved (Figures 4 and 5, Additional file 2: Table S3). Given these results, we suggest that HGTector is a useful addition to conventional BLAST-based approaches.

The HGTector pipeline has advantages of speed, automation, compatibility and low requirement for computational resources, making this program a generally applicable tool for discovery of vertical, and non-vertical history of genes, as well as initial HGT prediction. It is especially suitable for gaining a rapid and comprehensive overview of newly sequenced genomes to identify their evolutionary and ecological linkages with other organisms, facilitating further exploration of the functional drivers of the dynamics of genome evolution. It has to be made clear that an atypical BLAST hit distribution is an empirical observation, rather than a strict certification of HGT. Since an HGT predominantly reflects a past evolutionary event, it is theoretically impossible to identify exact gene donors and mechanisms, and any analysis is only an approximation to possible scenarios. Therefore, we recommend HGTector as a discovery tool for a detection of potential HGT-derived genes that can be further analyzed with phylogenetic approaches. This is much more effective approach than a very time-consuming and technically challenging process of a priori phylogenetic analysis of all genes within all target genomes, which becomes decreasingly feasible as more genomic data are present.

\section{Additional files}

\begin{abstract}
Additional file 1: Figure S1. Figure S1. Illustration of patterns of BLAST hit distribution and possible explanations. Figure S2. Precision-recall plot of results on simulated genomes. Figure S3. Relationship between global HGT rate and performance of methods. Figure S4. Fingerprints of genomes of various organisms. Figure S5. Distribution of BLAST hit weights of the $R$. felis genome. Figure S6. Heat map indicating percentages of predicted HGT-derived genes by putative bacterial donor groups in Rickettsia genomes. Figure S7. Heat map indicating percentages of predicted HGT-derived genes by functional annotations in Rickettsia genomes. Figure S8. Stability of results on the Rickettsia dataset with various simulated stochastic events. Figure S9. Stability of results on the Rickettsia dataset with simulated taxon sampling bias. Figure S10. Stability of results on the Rickettsia dataset with smaller sample size of genes for fingerprint calculation. Figure S11. Comparison of prediction results in the $R$. felis genome by multiple methods.
\end{abstract}

Additional file 2: Table S1. Protein accession numbers of predicted HGT-derived genes by HGTector. Table S2. Predicted HGT-derived genes categorized by gene orthology. Table S3. Prediction results of protein-coding genes in the R. felis genome by multiple methods. Table S4. Pairwise comparison of HGT-prediction results in the R. felis genome by multiple methods.

\section{Abbreviations}

BBH: Bidirectional best match; BLAST: Basic Local Alignment Search Tool; BLASTP: Protein BLAST; dbs: Density-based silhouette; GI: Genomic island; 
GO: Gene ontology; GUI: Graphical user interface; HGT: Horizontal gene transfer; HTML: Hypertext markup language; LPI: Lineage probability index; ML: Maximum likelihood; NCBI: National Center for Biotechnology Information; nr: Non-redundant protein sequences database; OF: Overlap factor; POE: Putative ORFans or annotation errors; SFG: Spotted fever group.

\section{Competing interests}

The authors declare that they have no competing interests.

\section{Authors' contributions}

QZ and KD conceived and designed the study. QZ developed the algorithm, wrote the program, performed the data analyses and drafted the manuscript. $\mathrm{KD}$ contributed to the design of algorithm and program, supervised the data analyses and edited the manuscript. MK contributed to the discussion and helped to revise the manuscript. All authors read and approved the final manuscript.

\section{Acknowledgements}

This research was funded by NSF DEB 1213740, awarded to KD.

\section{Author details}

${ }^{1}$ Department of Biological Sciences, University at Buffalo, State University of New York, 109 Cooke Hall, Buffalo, NY 14260, USA. ²Division of Vector-Borne Diseases, Centers for Disease Control and Prevention, 3156 Rampart Road, Fort Collins, CO 80521, USA.

Received: 11 May 2014 Accepted: 20 August 2014 Published: 26 August 2014

\section{References}

1. Ochman H, Lawrence JG, Groisman EA: Lateral gene transfer and the nature of bacterial innovation. Nature 2000, 405:299-304.

2. McDaniel LD, Young E, Delaney J, Ruhnau F, Ritchie KB, Paul JH: High frequency of horizontal gene transfer in the oceans. Science 2010, 330:50.

3. Abby SS, Tannier E, Gouy M, Daubin V: Lateral gene transfer as a support for the tree of life. Proc Natl Acad Sci U S A 2012, 109:4962-4967.

4. Andam CP, Williams D, Gogarten JP: Natural taxonomy in light of horizontal gene transfer. Biol Philos 2010, 25:589-602.

5. Doolittle WF: Phylogenetic classification and the universal tree. Science 1999, 284:2124-2129.

6. Delsuc F, Brinkmann $H$, Philippe $H$ : Phylogenomics and the reconstruction of the tree of life. Nat Rev Genet 2005, 6:361-375.

7. Simonson AB, Servin JA, Skophammer RG, Herbold CW, Rivera MC, Lake JA Decoding the genomic tree of life. Proc Natl Acad Sci U S A 2005, 102(Suppl 1):6608-6613.

8. Bapteste E, O'Malley MA, Beiko RG, Ereshefsky M, Gogarten JP, Franklin-Hall L, Lapointe F-J, Dupré J, Dagan T, Boucher Y, Martin W: Prokaryotic evolution and the tree of life are two different things. Biol Direct 2009, 4:34.

9. Koonin EV, Wolf Yl: The fundamental units, processes and patterns of evolution, and the tree of life conundrum. Biol Direct 2009, 4:33.

10. Lawrence JG, Ochman $\mathrm{H}$ : Amelioration of bacterial genomes: rates of change and exchange. J Mol Evol 1997, 44:383-397.

11. Doolittle WF, Boucher Y, Nesbø CL, Douady CJ, Andersson JO, Roger AJ: How big is the iceberg of which organellar genes in nuclear genomes are but the tip? Philos Trans R Soc Lond B Biol Sci 2003, 358:39-57. discussion 57-58.

12. Zhaxybayeva O, Doolittle WF: Lateral gene transfer. Curr Biol 2011, 21:R242-246.

13. Zhaxybayeva O: Detection and quantitative assessment of horizontal gene transfer. Methods Mol Biol 2009, 532:195-213.

14. Ragan MA, Harlow TJ, Beiko RG: Do different surrogate methods detect lateral genetic transfer events of different relative ages? Trends Microbiol 2006, 14:4-8.

15. Lawrence JG, Ochman $\mathrm{H}$ : Reconciling the many faces of lateral gene transfer. Trends Microbiol 2002, 10:1-4.

16. Koonin EV, Makarova KS, Aravind L: Horizontal gene transfer in prokaryotes: quantification and classification. Annu Rev Microbiol 2001, 55:709-742.

17. Altschul SF, Madden TL, Schäffer AA, Zhang J, Zhang Z, Miller W, Lipman DJ: Gapped BLAST and PSI-BLAST: a new generation of protein database search programs. Nucleic Acids Res 1997, 25:3389-3402.
18. Eisen JA: Assessing evolutionary relationships among microbes from whole-genome analysis. Curr Opin Microbiol 2000, 3:475-480.

19. Overbeek R, Fonstein M, D'Souza M, Pusch GD, Maltsev N: The use of gene clusters to infer functional coupling. Proc Natl Acad Sci U S A 1999, 96:2896-2901.

20. Nelson KE, Clayton RA, Gill SR, Gwinn ML, Dodson RJ, Haft DH, Hickey EK, Peterson JD, Nelson WC, Ketchum KA, McDonald L, Utterback TR, Malek JA, Linher KD, Garrett MM, Stewart AM, Cotton MD, Pratt MS, Phillips CA, Richardson D, Heidelberg J, Sutton GG, Fleischmann RD, Eisen JA, White O, Salzberg SL, Smith HO, Venter JC, Fraser CM: Evidence for lateral gene transfer between Archaea and bacteria from genome sequence of Thermotoga maritima. Nature 1999, 399:323-329.

21. Clarke GDP, Beiko RG, Ragan MA, Charlebois RL: Inferring genome trees by using a filter to eliminate phylogenetically discordant sequences and a distance matrix based on mean normalized BLASTP scores. J Bacterio/ 2002, 184:2072-2080.

22. Charlebois RL, Doolittle WF: Computing prokaryotic gene ubiquity: rescuing the core from extinction. Genome Res 2004, 14:2469-2477.

23. Dagan T, Artzy-Randrup Y, Martin W: Modular networks and cumulative impact of lateral transfer in prokaryote genome evolution. Proc Natl Acad Sci U S A 2008, 105:10039-10044.

24. Smillie CS, Smith MB, Friedman J, Cordero OX, David LA, Alm EJ: Ecology drives a global network of gene exchange connecting the human microbiome. Nature 2011, 480:241-244.

25. Sicheritz-Pontén T, Andersson SG: A phylogenomic approach to microbial evolution. Nucleic Acids Res 2001, 29:545-552.

26. Frickey T, Lupas AN: PhyloGenie: automated phylome generation and analysis. Nucleic Acids Res 2004, 32:5231-5238.

27. Charlebois RL, Clarke GD, Beiko RG, St Jean A: Characterization of species-specific genes using a flexible, web-based querying system. FEMS Microbiol Lett 2003, 225:213-220.

28. Podell S, Gaasterland T: DarkHorse: a method for genome-wide prediction of horizontal gene transfer. Genome Biol 2007, 8:R16.

29. Podell S, Gaasterland T, Allen EE: A database of phylogenetically atypical genes in archaeal and bacterial genomes, identified using the DarkHorse algorithm. BMC Bioinformatics 2008, 9:419.

30. Koski LB, Golding GB: The closest BLAST hit is often not the nearest neighbor. J Mol Evol 2001, 52:540-542.

31. Zhaxybayeva O, Nesbø CL, Doolittle WF: Systematic overestimation of gene gain through false diagnosis of gene absence. Genome Biol 2007, 8:402.

32. Willerslev E, Mourier T, Hansen AJ, Christensen B, Barnes I, Salzberg SL: Contamination in the draft of the human genome masquerades as lateral gene transfer. DNA Seq 2002, 13:75-76.

33. Stanhope MJ, Lupas A, Italia MJ, Koretke KK, Volker C, Brown JR: Phylogenetic analyses do not support horizontal gene transfers from bacteria to vertebrates. Nature 2001, 411:940-944.

34. Boto L: Horizontal gene transfer in evolution: facts and challenges. Proc Biol Sci 2010, 277:819-827.

35. Shokralla S, Spall JL, Gibson JF, Hajibabaei M: Next-generation sequencing technologies for environmental DNA research. Mol Ecol 2012, 21:1794-1805.

36. Le PT, Golaconda Ramulu H, Guijarro L, Paganini J, Gouret P, Chabrol O, Raoult D, Pontarotti P: An automated approach for the identification of horizontal gene transfers from complete genomes reveals the rhizome of Rickettsiales. BMC Evol Biol 2012, 12:243.

37. Merhej V, Notredame C, Royer-Carenzi M, Pontarotti P, Raoult D: The rhizome of life: the sympatric Rickettsia felis paradigm demonstrates the random transfer of DNA sequences. Mol Biol Evol 2011, 28:3213-3223.

38. Blanc G, Ogata H, Robert C, Audic S, Claverie J-M, Raoult D: Lateral gene transfer between obligate intracellular bacteria: evidence from the Rickettsia massiliae genome. Genome Res 2007, 17:1657-1664.

39. Ogata H, Renesto P, Audic S, Robert C, Blanc G, Fournier P-E, Parinello H, Claverie J-M, Raoult D: The genome sequence of Rickettsia felis identifies the first putative conjugative plasmid in an obligate intracellular parasite. PLOS Biol 2005, 3:e248.

40. Wolf Yl, Aravind L, Koonin EV: Rickettsiae and Chlamydiae: evidence of horizontal gene transfer and gene exchange. Trends Genet 1999, 15:173-175 
41. Weinert LA, Welch JJ, Jiggins FM: Conjugation genes are common throughout the genus Rickettsia and are transmitted horizontally. Proc Biol Sci 2009, 276:3619-3627.

42. R Core Team: R: A Language and Environment for Statistical Computing. Vienna, Austria: R Foundation for Statistical Computing; 2013.

43. Maddison DR, Swofford DL, Maddison WP: NEXUS: an extensible file format for systematic information. Syst Biol 1997, 46:590-621.

44. Gouy M, Guindon S, Gascuel O: SeaView version 4: a multiplatform graphical user interface for sequence alignment and phylogenetic tree building. Mol Biol Evol 2010, 27:221-224.

45. Tatusov RL, Koonin EV, Lipman DJ: A genomic perspective on protein families. Science 1997, 278:631-637

46. Fischer D, Eisenberg D: Finding families for genomic ORFans. Bioinformatics 1999, 15:759-762

47. Long M, Betrán E, Thornton K, Wang W: The origin of new genes: glimpses from the young and old. Nat Rev Genet 2003, 4:865-875

48. Brenner SE: Errors in genome annotation. Trends Genet 1999, 15:132-133.

49. Kohane IS, Hsing M, Kong SW: Taxonomizing, sizing, and overcoming the incidentalome. Genet Med 2012, 14:399-404.

50. Wong WC, Maurer-Stroh S, Eisenhaber F: More than 1,001 problems with protein domain databases: transmembrane regions, signal peptides and the issue of sequence homology. PLoS Comput Biol 2010, 6:e1000867.

51. Silverman BW: Using Kernel density estimates to investigate multimodality. J R Statist Soc B 1981, 43:97-99.

52. Silverman BW: Density Estimation. London: Chapman and Hall; 1986

53. Frederic I, Philippe G, Michele E: Pastecs: Package For Analysis Of Space-Time Ecological Series. R Package Version 1.3-13. 2013.

54. Kendall MG: Time-Series. London: Griffin; 1976.

55. Hartigan JA, Hartigan P: The dip test of unimodality. Ann Stat 1985. 13:70-84

56. Maechler M, Ringach D: Diptest: Hartigan's Dip Test Statistic For Unimodality-Corrected Code. In R Package Version 025-2. 2009

57. Menardi G: Density-based Silhouette diagnostics for clustering methods. Stat Comput 2010, 21:295-308.

58. Conesa A, Götz S, García-Gómez JM, Terol J, Talón M, Robles M: Blast2GO: a universal tool for annotation, visualization and analysis in functional genomics research. Bioinformatics 2005, 21:3674-3676.

59. Chen F, Mackey AJ, Stoeckert CJ Jr, Roos DS: OrthoMCL-DB: querying a comprehensive multi-species collection of ortholog groups. Nucleic Acids Res 2006, 34:D363-368.

60. Thompson JD, Higgins DG, Gibson TJ: CLUSTAL W: improving the sensitivity of progressive multiple sequence alignment through sequence weighting, position-specific gap penalties and weight matrix choice. Nucleic Acids Res 1994, 22:4673-4680.

61. Castresana J: Selection of conserved blocks from multiple alignments for their use in phylogenetic analysis. Mol Biol Evol 2000, 17:540-552.

62. Stamatakis A: RAxML-VI-HPC: maximum likelihood-based phylogenetic analyses with thousands of taxa and mixed models. Bioinformatics 2006, 22:2688-2690.

63. Rambaut A: FigTree 1.4. 2013. Available from http://tree.bio.ed.ac.uk/ software/figtree/.

64. Darriba D, Taboada GL, Doallo R, Posada D: jModelTest 2: more models, new heuristics and parallel computing. Nat Methods 2012, 9:772-772.

65. Merhej V, Raoult D: Rickettsial evolution in the light of comparative genomics. Biol Rev Camb Philos Soc 2011, 86:379-405.

66. Langille MGI, Brinkman FSL: IslandViewer: an integrated interface for computational identification and visualization of genomic islands. Bioinformatics 2009, 25:664-665.

67. Vitorino L, Chelo IM, Bacellar F, Zé-Zé L: Rickettsiae phylogeny: a multigenic approach. Microbiology 2007, 153:160-168.

68. Moreno-Hagelsieb G, Latimer K: Choosing BLAST options for better detection of orthologs as reciprocal best hits. Bioinformatics 2008, 24:319-324.

69. Hasan MS, Liu Q, Wang H, Fazekas J, Chen B, Che D: GIST: genomic island suite of tools for predicting genomic islands in genomic sequences. Bioinformation 2012, 8:203-205.

70. Hacker J, Kaper JB: Pathogenicity islands and the evolution of microbes. Annu Rev Microbiol 2000, 54:641-679.

71. Vernikos GS, Parkhill J: Interpolated variable order motifs for identification of horizontally acquired DNA: revisiting the Salmonella pathogenicity islands. Bioinformatics 2006, 22:2196-2203.
72. Hsiao W, Wan I, Jones SJ, Brinkman FSL: IslandPath: aiding detection of genomic islands in prokaryotes. Bioinformatics 2003, 19:418-420.

73. Waack S, Keller O, Asper R, Brodag T, Damm C, Fricke WF, Surovcik K, Meinicke P, Merkl R: Score-based prediction of genomic islands in prokaryotic genomes using hidden Markov models. BMC Bioinformatics 2006, 7:142.

74. Shrivastava S, Reddy CVSK, Mande SS: INDeGenIUS, a new method for high-throughput identification of specialized functional islands in completely sequenced organisms. J Biosci (Bangalore) 2010, 35:351-364.

75. Tu Q, Ding D: Detecting pathogenicity islands and anomalous gene clusters by iterative discriminant analysis. FEMS Microbiol Lett 2003, 221:269-275.

76. Che D, Hasan MS, Wang H, Fazekas J, Huang J, Liu Q: EGID: an ensemble algorithm for improved genomic island detection in genomic sequences. Bioinformation 2011, 7:311-314.

77. Biomatters: Geneious version 6.0. Available from http://www.geneious.com/

78. Popa O, Dagan T: Trends and barriers to lateral gene transfer in prokaryotes. Curr Opin Microbiol 2011, 14:615-623.

79. Schonknecht G, Chen WH, Ternes CM, Barbier GG, Shrestha RP, Stanke M, Brautigam A, Baker BJ, Banfield JF, Garavito RM, Carr K, Wilkerson C, Rensing SA, Gagneul D, Dickenson NE, Oesterhelt C, Lercher MJ, Weber AP: Gene transfer from bacteria and archaea facilitated evolution of an extremophilic eukaryote. Science 2013, 339:1207-1210.

80. Langille MGI, Hsiao WWL, Brinkman FSL: Evaluation of genomic island predictors using a comparative genomics approach. BMC Bioinformatics 2008, 9:329.

81. Philippe H, Brinkmann H, Lavrov DV, Littlewood DTJ, Manuel M, Wörheide G, Baurain D: Resolving difficult phylogenetic questions: why more sequences are not enough. PLOS Biol 2011, 9:e1000602.

doi:10.1186/1471-2164-15-717

Cite this article as: Zhu et al.: HGTector: an automated method facilitating genome-wide discovery of putative horizontal gene transfers. BMC Genomics 2014 15:717.

\section{Submit your next manuscript to BioMed Central and take full advantage of:}

- Convenient online submission

- Thorough peer review

- No space constraints or color figure charges

- Immediate publication on acceptance

- Inclusion in PubMed, CAS, Scopus and Google Scholar

- Research which is freely available for redistribution

Submit your manuscript at www.biomedcentral.com/submit
C Biomed Central 